\title{
Filter Intolerable Posts from OSN User Walls
}

\author{
Zahraa Modher Nabat ${ }^{1}$, Hemant Mahajan ${ }^{2}$ \\ ${ }^{1}$ Modern College of Arts, Science and Commerce, Pune \\ ${ }^{2}$ Director and Research Analysis, Godwit Technologies Pvt. Ltd
}

\begin{abstract}
As we know, these days everyone seems to be victimization On-line Social Networks (OSNs) to speak and share data. Therefore, one vital want in these days On-line Social Networks (OSNs) is to offer users the power to manage the messages denote on their own personal area to avoid that unwanted content is displayed. on-line informal communication (OSN's) has was a standout amongst the foremost current intelligent medium to impart, impart and disperse the human life knowledge. therefore, the increasing utilization of it incorporates providing of substance like free messages, pictures, sounds and options. that currently and once more isn't vulnerable to be imparted on user's personal divider. For the nowadays OSNs have given a little backing to the present. For this reason, to be increased, we've got projected a framework that offers the OSN purchasers a right away management on such type of messages. This can be achieved through a versatile rule-based system that enables users to customize the filtering criteria to be applied to their walls, Machine Learning-based soft classifier mechanically labeling messages in support of content-based filtering. Online informal communication (OSN's) has was a standout amongst the foremost current intelligent medium to impart, impart and disperse the human life knowledge. Therefore, the increasing utilization of it incorporates providing of substance like free messages, pictures, sounds and options. that currently and once more isn't vulnerable to be imparted on user's personal divider. For the nowadays OSNs have given a little backing to the present. For this reason, to be increased, we've got projected a framework that offers the OSN purchasers a right away management on such type of messages. This could be accomplished utilizing a principle primarily based framework that allows purchasers to use separating criteria on their own personal divider. It likewise utilizes a machine learning primarily delicate classifier consequently marking messages in backing of substance based winnowing, list terms on line social organizations and knowledge divider.
\end{abstract}

Keywords: Social networking, Unwanted massages, filtering OSN user wall

\section{Introduction}

One noteworthy issue in today's on-line Social Networks (OSNs) is to present purchaser's skills to manage the messages denote on their lonesome individual house and to remain far from that undesirable data being shown. during this proposition, we have a tendency to propose a framework that enables OSN purchasers to possess an instantaneous management on the messages denote on their dividers. It's accomplished by applying separation criteria on the dividers. Associate in Nursing social organization incorporates personal informing, speak workplace and record or photograph giving capacities. Purchasers of those locales will specific their thoughts and views moreover will die them on their dividers also. This divider is Associate in Nursing open house therefore others will likewise see what has been composed on one's divider. Consequently, in OSN there's believability of posting awful or undesirable messages on divider that is apparent to others also. to handle this issue, we've got projected a framework that channels such type of messages and this can be finished with the desire of the wall's holder thus the aim of the current work is to propose and by experimentation value an automatic system, referred to as Filtered Wall (FW), able to filter unwanted messages from OSN user walls. The support for content based mostly user preferences is that the key plan of projected system. this can be potential give thanks to the employment of a Machine Learning (ML) text categorization procedure [12] able to mechanically assign with every message a group of classes supported its content. Section II reviews connected work, whereas Section III presents the abstract design of the projected system. Section IV describes the assembled text classification technique accustomed reason text contents, whereas Section V explains FRs and BLs. Section VI describes the case study. Finally, section VII concludes the paper. This divider is
Associate in Nursing open house therefore others will likewise see what has been composed on one's divider. Consequently, in OSN there's believability of posting awful or undesirable messages on divider that is apparent to others also. to handle this issue, we've got projected a framework that channels such type of messages and this can be finished with the desire of the wall's holder.

In explicit, we have a tendency to base the general short text classification strategy on Radial Basis perform Networks (RBFN) for his or her proved capabilities in acting as soft classifiers, in managing clamant knowledge and in and of itself obscure categories. Moreover, the speed in playing the educational part creates the premise for Associate in Nursing adequate use in OSN domains, also as facilitates the experimental analysis tasks.

\section{Literature Survey}

Filtering is predicated on explanations of individual or cluster data preferences that usually represent long interests. Users get solely the info that's extracted. data filtering systems are meant to reason a stream of dynamically generated data and gift it to the user that data that are possible to satisfy user necessities.

[1] A. Adomavicius and G. Tuzhilin, presents an outline of the sphere of recommender systems and describes this generation of advice ways that are typically classified into the subsequent 3 main categories: content-based, cooperative, and hybrid recommendation approaches. This paper conjointly describes varied limitations of current recommendation ways and discusses doable extensions that may improve recommendation capabilities and build recommender systems applicable to a good broader vary of applications.

\section{Volume 5 Issue 4, April 2016}




\section{International Journal of Science and Research (IJSR) \\ ISSN (Online): 2319-7064 \\ Index Copernicus Value (2013): 6.14 | Impact Factor (2015): 6.391}

[2] M. Chau and H. Chen, because the net continues to grow, it's become progressively tough to go looking for relevant data victimization ancient search engines. Topicspecific search engines give another thanks to support economic data retrieval on the net by providing additional precise and customized looking out in varied domains. However, developers of topic-specific search engines ought to address 2 issues: the way to find relevant documents (URLs) on the net and the way to separate digressive documents from a group of documents collected from the net. This paper reports our analysis in addressing the second issue. We tend to propose a machine-learning-based approach that mixes web page analysis and net structure analysis.

[3] R.J. Mooney and L. Roy, recommender systems improve access to relevant products and data by creating personalized suggestions supported previous samples of a user's likes and dislikes. Most existing recommender systems use cooperative filtering ways that base recommendations on alternative users' preferences. Against this, content-based ways use data regarding AN Item itself to form suggestions. This approach has the advantage of having the ability to suggest antecedently unrated things to users with distinctive interests and to supply explanations for its recommendations. We tend to describe a content-based book recommending system that utilizes data extraction and a machine learning formula for text categorization. Initial experimental results demonstrate that this approach will manufacture correct recommendations.

[4] F. Sebastiani, the automatic categorization (or classification) of texts into predefined classes has witnessed a booming interest within the last ten years, attributable to the accrued accessibility of documents in digital kind and therefore the succeeding ought to organize them. within the analysis community the dominant approach to the current downside is predicated on machine learning techniques: a general inductive method mechanically builds a classifier by learning, from a group of reclassified documents, the characteristics of the classes. the benefits of this approach over the data engineering approach (consisting within the manual definition of a classifier by domain experts) arean awfully smart effectiveness, significant savings in terms of skilled labor power, and simple movability to completely different domains. This survey discusses the most approaches to text categorization that fall inside the machine learning paradigm. we'll discuss thoroughly problems touching on 3 completely different issues, namely, document illustration, classifier construction, and classifier analysis.

[5] J. Golbeck, Social networks are a well-liked movement on the net. On the linguistics net, it's straightforward to form trust annotations to social relationships. during this paper, we tend to gift a 2 level approach to desegregation trust, provenance, and annotations in linguistics net systems. we tend to describe AN formula for inferring trust relationships victimization source data and trust annotations in linguistics Web-based social networks. Then, we tend to gift AN application, Film Trust that mixes the computed trust values with the source of alternative annotations to change the web site. The Film Trust system uses trust to calculate personalizedrecommended movie ratings and to order reviews.

\section{Modules to be Implemented}

\subsection{Pre-processing}

The Fig. 3.1 shows the first aim of the pre-processing part is to get rid of from the input message all characters and terms that may probably have an effect on the standard of cluster descriptions.

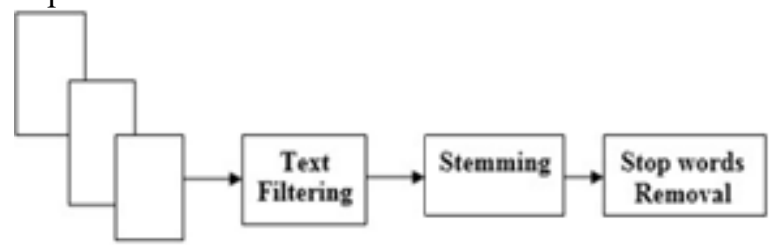

Figure 3.1: Pre-processing of Message

\section{Pre-processing steps}

/** section 1: Pre-processing **/

for each document determine the document's language; apply stemming; mark stop words; $\}$

There are 3 steps to the preprocessing phase: Text filtering, Stemming and Stop words marking.

\section{a) Text Filtering}

In the text filtering step, all terms that are useless or would introduce noise in filtering process are removed from the input message. Among such terms are:

- HTML tags (e.g. <table >) and entities (e.g. \&) if any.

- Non-letter characters such as \$”, \%" or \#” (except white spaces and sentence markers such as .', ?' or !') Note that at this stage the stop-words are not removed from the input.

\section{b) Stemming}

Stemming algorithms area unit accustomed remodel the words in texts into their grammatical root type, and area unit chiefly accustomed improve the data Retrieval Systems potency.

To stem a word is to cut back it to an additional general type, presumably its root. for instance, stemming the term fascinating might manufacture the term interest. Although the stem of a word may not be its root, we would like all words that have constant stem to own constant root.

\section{c) Elimination of Stop Words}

After stemming it is necessary to remove unwanted words. There are 400 to 500 types of stop words such as off, and, the, etc., that provide no useful information about the message. Stop-word removal is the process of removing these words. Stop-words account for about $20 \%$ of all words in a typical document. 


\section{International Journal of Science and Research (IJSR) \\ ISSN (Online): 2319-7064}

Index Copernicus Value (2013): 6.14 | Impact Factor (2015): 6.391

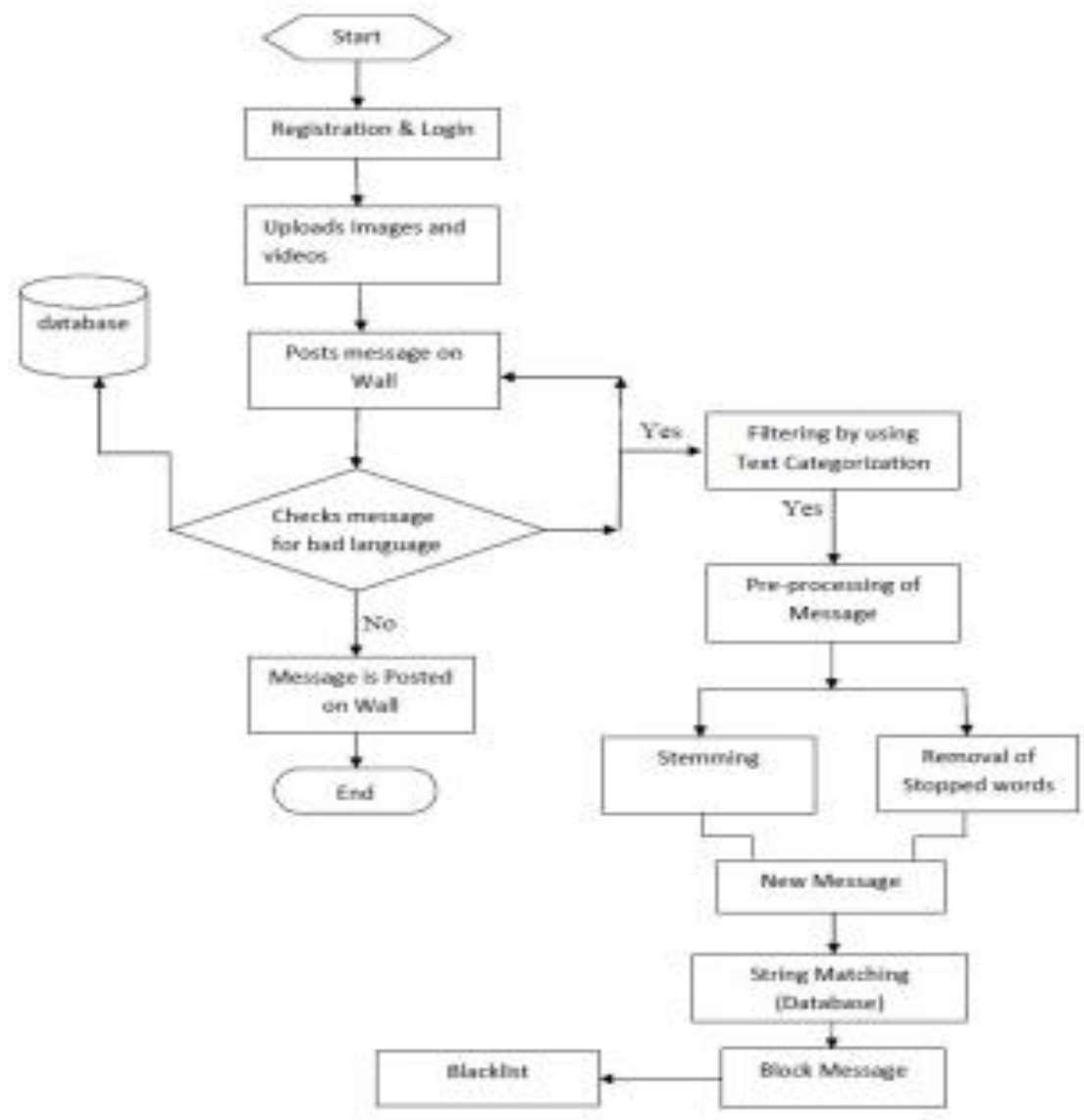

Figure 3.2: Data Flow of the System

\subsection{Mathematical Model}

\section{a) For Filtering Rules}

\section{Input}

Filtering Rules are customizable by the user. User can have authority to decide what contents should be blocked or displayed on his wall by using Filtering rules. For specify a Filtering rules user profile as well as user social relationship will be considered. $\mathrm{FR}=\{$ Trustier, SOUs, Rule, $\mathrm{TuV}\} \mathrm{FR}$ is dependent on following factors

- Trustier

- Set of Users (SOUs)

- Rule

- Action

Trustier is a person who defines the rules.

SOUs denote the set of OSN user.

Rule is a Boolean expression defined on content.

\section{Process}

$\mathrm{FM}=\{$ SOUs, Rule $==$ category (Violence, Vulgar, offensive, Hate, Sexual), TuV\}

- FM

- SOUs

- Rule

- $\mathrm{TuV}$

Here, FM Block Messages at basic level. SOUs Denotes set of users Rule class of nominative contents in message.
$\mathrm{TuV}$ is that the trust price of sender. In process, when giving input message, the system can compare the text with the various classes that square measure prevented. If message found therein prevented variety of class then message can show to the user that ean't send this sort of messages", and still the user desires to send the message he/she will continue with causation the message. The Trustier, WHO gets the message, however the words that square measure defended within the rule square measure sent in $* * * *$ format. when obtaining the message, the Trustier can provide the Feedback (FB) to the sender and also the sender can gain the $\mathrm{TuV}$ consequently. method denotes the action to be performed by the system on the messages matching Rule and created by users known by SOUs.

E.g. $\mathrm{FM}==$ Friends, Rule $==$ category (Vulgar, Sexual), $\mathrm{TuV}>50$

i.e. Trustier can settle for the message from friends however message mustn't contain vulgar or sexual words. Message containing such words can have an effect on the $\mathrm{TuV}$ of sender. currently the question arises, calculation of $\mathrm{TuV}$.

\section{Trust Value Calculations}

Trust price of any user in OSN relies on the feedback they gain by the user to whom they sent a message. Feedback from the user should even be trust worthy. That's why the FB is categorized into following: -

- Positive with content (PC) - sensible FB, message is suitable with objectionable content. this may increase the $\mathrm{TuV}$ of sender. 


\section{International Journal of Science and Research (IJSR) \\ ISSN (Online): 2319-7064}

Index Copernicus Value (2013): 6.14 | Impact Factor (2015): 6.391

- Positive while not content (PWC) - sensible FB, message is suitable as this message doesn't contain objectionable content. This may increase the TuV of sender.

- Negative with content (NC) - unhealthy FB, such messages should not be sent once more, which are against the Rule. This may decrease the TuV of sender.

- Negative while not content (NWC) - unhealthy FB, message doesn't contain any objectionable content however the Trustier is giving negative FB. Such style of FB from Trustier can have an effect on the TuV of its own, and also the TuV of sender can stay same. So, supported on top of classes the $\mathrm{TuV}$ are going to be calculated as follows: -

- $\mathrm{FB}$ as one and a couple of $\mathrm{TuV}=\mathrm{TuV}+\mathrm{abs}[(\mathrm{PC}+\mathrm{PWC})$ / (NC+NWC)]

- $\mathrm{FB}$ as three $\mathrm{TuV}=\mathrm{TuV}-[1+(\mathrm{NC}+\mathrm{NWC}) /(\mathrm{PC}+\mathrm{PWC})]$ for $[(\mathrm{NC}+\mathrm{NWC}) /(\mathrm{PC}+\mathrm{PWC})]<$ one

Otherwise, send system generated message to sender, FB Negative with content exceeds limit of Threshold worth (ThV) and deduct five points from $\mathrm{TuV}$, therefore $\mathrm{ThV}=\mathrm{TuV}-5$. FB as four $\mathrm{TuV}=\mathrm{TuV}$ of sender, however $\mathrm{TuV}=\mathrm{TuV}-[1+(\mathrm{NC}+\mathrm{NWC}) /(\mathrm{PC}+\mathrm{PWC})]$ for Trustier.

\section{Output}

$\mathrm{PFM}=$ Rule, $\mathrm{M} / / \mathrm{Y}$

- PFM Percentages of filtered message in a year or month. In general, more than a filtering rule can apply to the same user. A message is therefore published only if it is not blocked by any of the filtering rules that apply to the message creator.

\section{b) Blacklists}

BLs is directly managed by the system. This should be able to determine the users to be inserted in the BL and decide when to retain user back from the BL. To enhance flexibility, such information is given to the system through a set of rules, hereafter called BL rules.

\section{BL rules}

INPUT $=$ Sender, FB, TuV, ThV Where

- Sender is the OSN user who is sending the message;

- FB is the FeedBack gain by the sender after sending the message.

- $\mathrm{TuV}$ is the new Trust Value calculated as formulas specified in A.3.

- ThV is the Threshold Value.

\section{BL Rules}

$\mathrm{ThV}=\mathrm{PC}+\mathrm{PWC}$ when, $\mathrm{PC}+\mathrm{PWC}=\mathrm{NC}+\mathrm{NWC}$.

For sender, when 5 points are deducted by system, which means sender cross the $\mathrm{ThV}$ put sender into $\mathrm{BL}$ for a specific duration. For Trustier, after giving feedback, check $\mathrm{ThV}$, if true, put Trustier in BL for specific duration.

\section{Work Done}

In this section we are discussing the practical environment, scenarios, performance metrics used etc.

\subsection{Input}

In this Training and Testing Image is the input for our practical experiment.

\subsection{Hardware Requirements}

Processor:Pentium IV 2.6 GHz

Ram:512 Mb

Hard Disk:20 GB

\subsection{Software Requirements:}

Front End:J2SE

Back End:MySQL 5.1

Tools Used: Net Beans 7.2.1 or above

Operating System: Windows 7/8

\section{Results of Practical Work}

Following figures are showing results for practical work which is done. Following figure showing the main screen. Post Undesired Message on other user Wall.

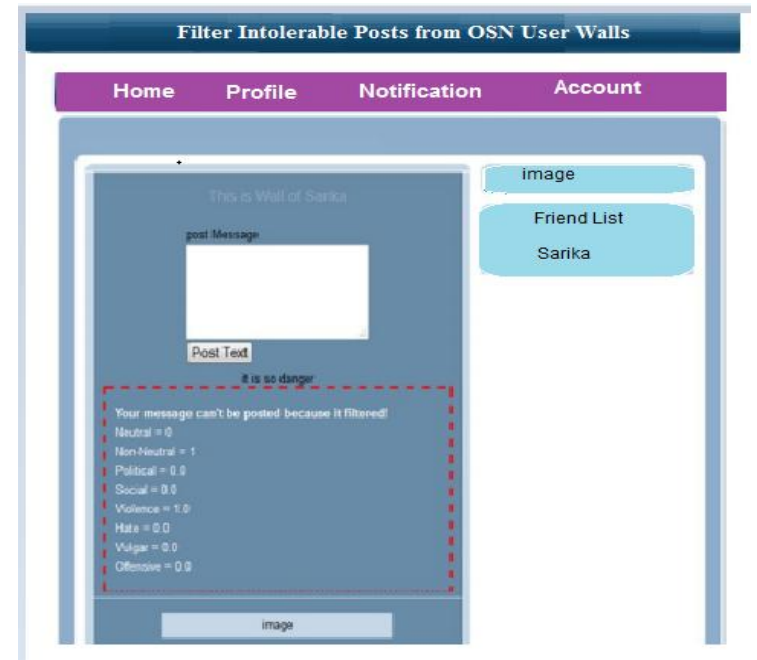

Figure 5.1: Post Undesired Message on other user Wall

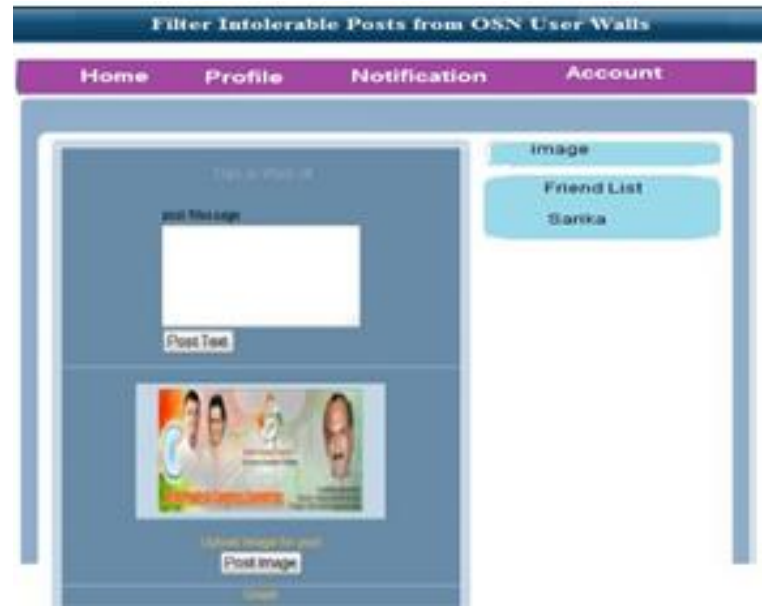

Figure 5.2: Warning to misbehavior user block 


\section{International Journal of Science and Research (IJSR) \\ ISSN (Online): 2319-7064}

Index Copernicus Value (2013): 6.14 | Impact Factor (2015): 6.391

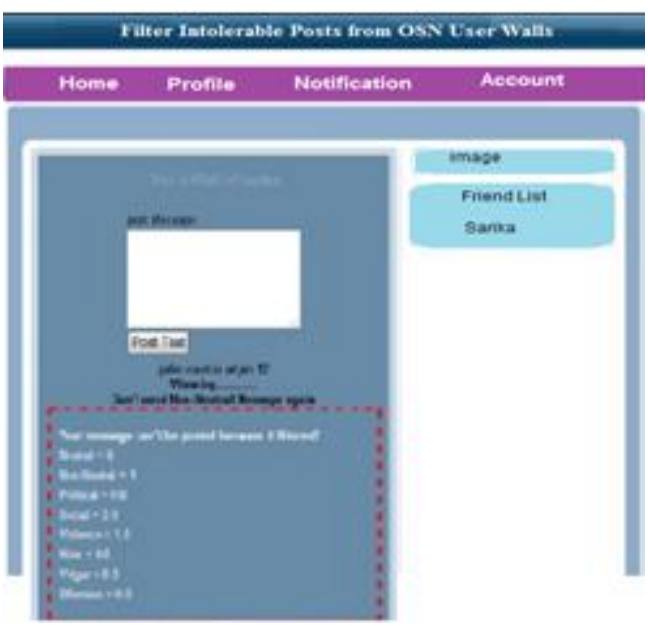

Figure 5.3: Post Undesired Image on other user Wall

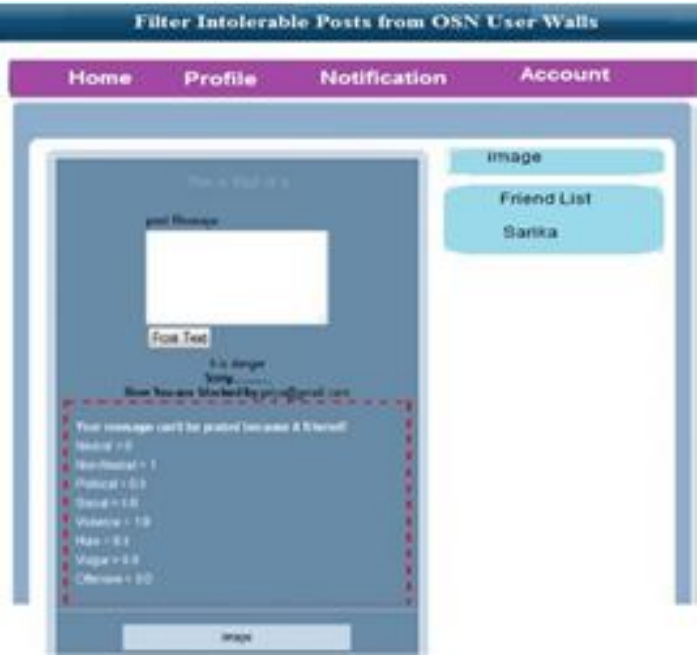

Figure 5.4: Permission Blocking misbehavior use

\section{Conclusion and Future Work}

In this paper, filter intolerable post from OSN wall is presented. The first step of the project is to classify the content using several rules. next step is to filter the undesired rules. Finally blacklist rule is implemented. so that owner of the user can insert the user who posts undesired messages. Better privacy is given to the OSN wall using our system.

\section{References}

[1] N. J. Belkin and W. B. Croft, Information filtering and information retrieval: Two sides of the same coin?" Communications of the ACM, vol. 35, no.12, pp. 2938, 1992.

[2] P. W. Foltz and S. T. Dumais, Personalized information delivery: An analysis of information filtering methods," Communications of the ACM, vol. 35 , no. 12 , pp. 51-60, 1992.

[3] P. E. Balance, Competitive agents for information filtering," Communications of the ACM, vol. 35, no. 12, p. 50, 1992.

[4] F. Sebastiani, Machine Learning in Automated Text Categorization," ACM Computing Surveys, vol. 34, no. 1, pp. 1-47, 2002.
[5] M. Vanetti, E. Binaghi, B. Carminati, M. Carullo, and E. Ferrari, -Cotent-Based Filtering in On-Line Social Networks," Proc. ECML/PKDD Workshop Privacy and Security Issues in Data Mining and Machine Learning (PSDML '10), 2010.

[6] N.J. Belkin and W.B. Croft, Information Filtering and Information Retrieval: Two Sides of the Same Coin?" Comm. ACM, vol. 35, no. 12, pp. 29-38, 1992.

[7] P.J. Denning, Electronic Junk," Comm. ACM, vol. 25, no. 3, pp. 163-165, 1982.

[8] P.W. Foltz and S.T. Dumais, Personalized Information Delivery: An Analysis of Information Filtering Methods," Comm. ACM, vol. 35, no. 12, pp. 51-60, 1992.

[9] P.S. Jacobs and L.F. Rau, Scissor: Extracting Information from Online News," Comm. ACM, vol. 33, no. 11, pp. 88-97, 1990.

[10] S. Pollock, A Rule-Based Message Filtering System,' ACM Trans. Office Information Systems, vol. 6, no. 3, pp. 232-254, 1988.

[11]P.E. Baclace, Competitive Agents for Information Filtering," Comm. ACM, vol. 35, no. 12, p. 50, 1992.

[12] P.J. Hayes, P.M. Andersen, I.B. Nirenburg, and L.M. Schwandt, Fics: A Shell for Content-Based Text Categorization," Proc. Sixth IEEE Conf. Artificial Intelligence Applications (CAIA '90), pp. 320-326, 1990.

[13] G. Amati and F. Crostini, - Pobabilistic Learning for Selective Dissemination of Information," Information Processing and Management, vol. 35, no. 5, pp. 633654, 1999.

[14] M.J. Azzanni and D. Bills us, Eearning and Revising User Profiles: The Identification of Interesting Web Sites," Machine Learning, vol. 27, no. 3, pp. 313-331, 1997.

[15]Y. Zhang and J. Callan, Maximum Likelihood Estimation for Filtering Thresholds," Proc. 24th Ann. Int'1 ACM SIGIR Conf. Research and Development in Information Retrieval, pp. 294-302, 2001.

[16] C. Pate, F. Damara, S.M. Weiss, D. Shalom, and M. Weiss, - Atomated Learning of Decision Rules for Text Categorization," Trans. Information Systems, vol. 12, no. 3, pp. 233-251, 1994.

[17] S. Dumais, J. Platt, D. Heckerman, and M. Sahami, Inductive Learning Algorithms and Representations for Text Categorization," Proc. Seventh Int'l Conf. Information and Knowledge Management (CIKM '98), pp. $148-155,1998$

[18]A. Cagayan, M. Snoras, J. Jacoby, J. Maze, R. Jones, and K. Kumar, - Earn Sesame-A Learning Agent Engine," Applied Artificial Intelligence, vol. 11, pp. 393-412, 1997.

[19] S. Chaudhury and U. Dayal, -AnOverview of Data Warehousing and OLAP Technology," ACM SIGMOD Record, vol. 26, no. 1, pp. 65-74, 1997.

[20] Y.-H. Chien and E.I. George, -ABayesian Model for Collaborative Filtering," Proc. Seventh Int'l Workshop Artificial Intelligence and Statistics, 1999.

[21] M. Claypool, A. Goshala, T. Miranda, P. Murnion, D. Nets, and M. Sarton, Combining Content-Based and Collaborative Filters in an Online Newspaper," Proc. ACM SIGIR '99 Workshop Recommender Systems: Algorithms and Evaluation, Aug. 1999. 


\section{International Journal of Science and Research (IJSR) \\ ISSN (Online): 2319-7064}

Index Copernicus Value (2013): 6.14 | Impact Factor (2015): 6.391

[22] W.W. Cohen, R.E. Schapiro, and Y. Singer, - Earning to Order Things," J. Artificial Intelligence Research, vol. 10, pp. 243-270, 1999.

[23]D. Cohn, L. Atlas, and R. Ladner, Improving Generalization with Active Learning," Machine Learning, vol. 15, no. 2, pp. 201-221, 1994.

[24]D. Cohn, Z. Ghahramani, and M. Jordan, - Ative Learning with Statistical Models," J. Artificial Intelligence Research, vol. 4, pp. 129- 145, 1996. 